\title{
Demand Flexibility Potential Model for Multi-Zone Commercial Buildings using Internal HVAC System States
}

\author{
Elvin Vindel \\ Carnegie Mellon University \\ Pittsburgh, PA, USA \\ evindel@cmu.edu \\ Burcu Akinci \\ Carnegie Mellon University \\ Pittsburgh, PA, USA \\ bakinci@cmu.edu
}

\author{
Mario Bergés \\ Carnegie Mellon University \\ Pittsburgh, PA, USA \\ marioberges@cmu.edu \\ Olga Kavvada \\ ENGIE Lab CRIGEN \\ Paris, France \\ olga.kavvada@engie.com
}

\begin{abstract}
The rapidly changing outlook of the electric grid with growing use of renewable energy imposes additional needs for operational flexibility. An opportunity to fulfill a portion of these grid services can come from the demand flexibility (DF) of commercial buildings. However, the practicality using these systems as grid resources hinges on the development of scalable and accurate load flexibility models. To satisfy both properties, we propose using internal HVAC system states to generate a feature called thermal unloading potential to predict demand flexibility. The proposed feature is designed to capture the balance between internal and external thermal loading. We evaluate this DF estimation framework with simulation experiments using three standard prototype office buildings during cooling operation with five distinct weather scenarios, yielding over 15,000 load shedding events. Subsequently, we fit regression models using our proposed feature and compare it to models that rely on outdoor air temperature (OAT) as predictor variable. Our results show that overall the proposed feature has a $55 \%$ reduction in error on average across all simulated events.
\end{abstract}

\section{CCS CONCEPTS}

- Hardware $\rightarrow$ Smart grid; Energy generation and storage; • General and reference $\rightarrow$ Empirical studies.

\section{KEYWORDS}

demand flexibility, grid-interactive efficient buildings, HVAC systems, grid services

\section{ACM Reference Format:}

Elvin Vindel, Mario Bergés, Burcu Akinci, and Olga Kavvada. 2021. Demand Flexibility Potential Model for Multi-Zone Commercial Buildings using Internal HVAC System States. In ACM International Conference on Systems for Energy-Efficient Built Environments (BuildSys '21), November 17-18, 2021, Coimbra, Portugal. ACM, New York, NY, USA, 4 pages. https://doi.org/10. $1145 / 3486611.3486654$

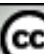

This work is licensed under a Creative Commons Attribution International 4.0 License. BuildSys '21, November 17-18, 2021, Coimbra, Portugal

(c) 2021 Copyright held by the owner/author(s).

ACM ISBN 978-1-4503-9114-6/21/11.

https://doi.org/10.1145/3486611.3486654

\section{INTRODUCTION}

As the electric grid modernizes to include large shares of variable generation, new sources of operational flexibility are essential to support energy affordability and reliability [23]. The deployment of grid-interactive technologies in buildings stands out as a valuable resource to provide these essential grid services [22]. In this work, we focus on commercial buildings whose electricity consumption accounts for about $40 \%$ of the annual total use in the US [24]. The most prevalent form of demand flexibility in commercial buildings comes from non-automated peak reductions, however this approach will be of limited value in a smart grid future [1]. Instead, valuable demand flexibility products will include generation capacity, contingency reserves, and ramping, which generally have stricter contractual requirements [3]. Recent reports ([6]) highlight the low performance compliance of demand response (DR) resources, thereby complicating the case for buildings as an adequate grid resource. Hence, the accurate estimation of demand flexibility potential (i.e., a load flexibility model) can improve operational efficiency, support planning/design decisions, and ensure accurate payments to resources [14].

The objective of a load flexibility model, for a participating gridinteractive building, is to inform the operator (or aggregator) its predicted flexibility for an upcoming event [21]. We narrow the application to models that predict the magnitude of a load shed event $(\Delta P)$ actuated through variable-air-volume (VAV) systems of heating, ventilation and air conditioning (HVAC) loads [16]. VAVs are commonly used in commercial buildings and often used as reference systems to evaluate new technologies [17]. However, the diversity in composition, size, and performance of these systems makes it challenging to subscribe to a universal modeling approach. This observation gives rise to two, often conflicting, challenges in identifying models for HVAC-based demand flexibility in commercial buildings: accuracy and scalability. On one hand, the accuracy requirements are tied to the contractual requirements of reliability services. As an example, a contingency reserve resource can be contractually obligated to deploy and sustain at least $95 \%$ of a committed deviation [9]. On the other hand, the need for a scalable solution stems from the fact that demand flexibility is dependent on the widespread adoption from buildings [18]. This implies the need for models that greatly reduce the complexity of these systems and are general enough to be deployed across a diverse fleet of existing buildings [10]. 
In this work, we focus on the intersection of these two challenges by proposing the thermal unloading potential feature $\left(\alpha_{u}\right)$, derived from HVAC states, to use as a predictor variable of load shedding potential. The contributions of our work are the following. First, the proposed feature captures the balance between internal and external thermal loading inherent to the installed HVAC systems. Second, existing approaches commonly identify independent models specific to the time of day, while ours proposes one model per building. Third, our approach improves the accuracy of load shedding potential when compared to existing methods that rely on only outdoor air temperature.

\section{RELATED WORK}

The development of load flexibility models for commercial buildings has its roots in peak demand reduction programs [19]. This led to findings that suggested that load shedding potential generally has a positive correlation with OAT [15]. Additionally, global thermostat reset (GTR) emerged as a scalable candidate to actuate demand flexibility events in buildings due to its simplicity [15]. Field studies continued to reinforce both the choice of control actuation and connection to OAT [18, 27]. In this same line of work, [26] developed a demand flexibility estimation framework to identify models for each hour of the day. A comprehensive analysis of 36 buildings in California suggested, counterintuitively, the possibility of a negative correlation between OAT and load shed potential [20]. However, recent work is evidence that the approach continues to be at the forefront of research and practice [11]. Other works have proposed demand flexibility quantification methods using models that incorporate information about the building and its HVAC systems. For example, [12] proposed a demand flexibility model that takes into account the building's energy physics and comfort constraints. In [5], demand flexibility strategies were tested on a dynamic VAV system model revealing that terminal damper positions were significant in determining performance. Considering terminal damper position properties, [10] proposed a low-complexity model to predict load shedding potential actuated through a GTR. Our model builds off of the principles in a previous study that connected terminal damper positions to the power consumption of the supply fan [25]. The difference is that here we include the power consumption of all HVAC components (supply/return fans, chiller, and pumps), extend the model to multiple independent HVAC systems in one building, and the application is to load shedding events exclusively. We compare our approach to those that use OAT as a predictor variable given that it is the most prevalent approach and has a strong background of field demonstrations [13, 15, 18-20, 26].

\section{APPROACH}

Our goal is to develop a model that incorporates HVAC system states to predict load shedding potential when actuated through a GTR. Section 3.1 elaborates on how terminal damper positions can be used as a proxy for the thermal loading state of a building. This is followed by Section 3.2 which describes how we use this variable to compute the thermal unloading potential. A visual summary of our approach can be seen in Figure 1.

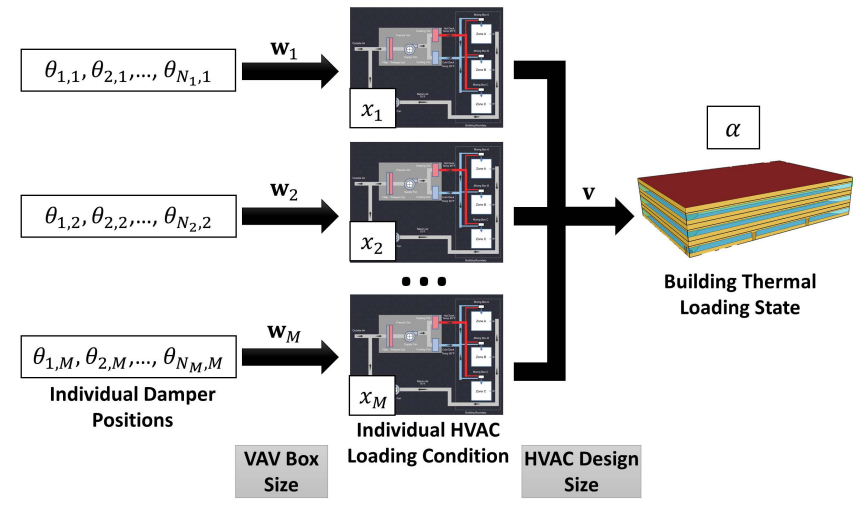

Figure 1: Proposed feature: thermal loading state $(\alpha)$ using damper positions $\theta_{i, j}$ terminal box $i$ in HVAC system $j$.

\subsection{Building Thermal Loading State}

Consider a commercial building that has $M$ independent VAV systems of $N_{j}$ terminal boxes each. For each VAV system $(j)$ we denote the vector of steady-state terminal damper positions as $\boldsymbol{\theta}_{\boldsymbol{j}}=$ $\left\{\theta_{1, j}, \theta_{2, j}, \ldots, \theta_{N_{j}, j}\right\} \in \mathbb{R}^{N_{j}}$. During operation, these positions adapt to thermal loads over time $(t)$, but for simplicity we will drop the temporal identifier. Each $\theta_{i, j} \in\left[\bar{\theta}_{i, j}, 1\right]$ is the damper position with a lower limit dictated by minimum ventilation requirements. We define a single system loading state $x_{j} \in \mathbb{R}$ by taking terminal damper positions $\theta_{j}$ multiplying them by proportional weights obtained from the terminal box sizes $\mathbf{w}_{j} \in \mathbb{R}^{N_{j}}$ as shown in Equation 1. For further details see [25].

$$
x_{j}=\mathbf{w}_{j}^{\top} \theta_{j}
$$

To account for multiple independent HVAC systems with different sizes, we propose to use the system design size $\mathbf{d} \in \mathbb{R}^{M}$ (i.e., supply fan size in CFM). Similar to the previous step, we compute corresponding proportional weights $\mathbf{v}=\frac{\mathbf{d}}{\sum_{k} d_{k}}$. Next, we form a vector containing individual HVAC system loading states $\mathbf{x}=\left\{x_{1}, x_{2}, \ldots, x_{M}\right\} \in \mathbb{R}^{M}$. Finally, we can compute a single state variable $\alpha$ which we call the thermal loading state as shown in Equation 2. In simple terms, this state variable captures the loading conditions experienced by all thermal zones into a single value. More importantly, the upper and lower limits observed using historical data are an indication of the operational range of all the installed HVAC systems. Note that because the damper positions are time varying, $\alpha(t)$ can be computed from the values at time $t$.

$$
\alpha=\mathbf{v}^{\top} \mathbf{x}
$$

\subsection{Demand Flexibility Model}

Our proposed model leverages the information captured by the thermal loading state to predict load shedding potential $(\Delta P)$. The first assumption is that after a building is actuated for a load shedding event (e.g., $+2^{\circ} \mathrm{F}$ GTR), terminal boxes react to the sudden change in zone setpoint by reaching their minimum ventilation limits (i.e., $\bar{\theta}_{i, j}$ ). Using historical data and calculating the sample minimum for every damper we can compute a corresponding $\bar{\alpha}$ for 

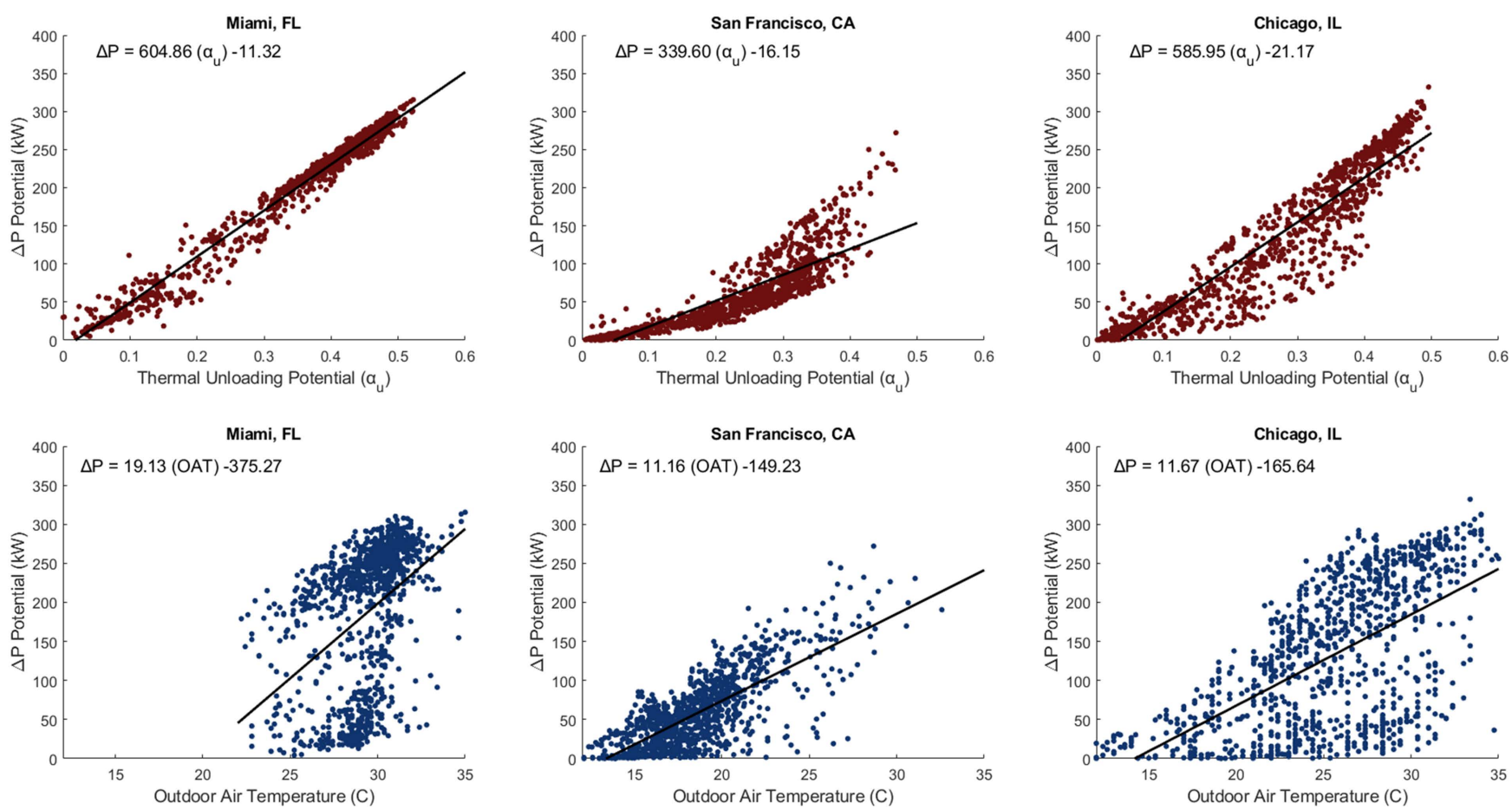

Figure 2: Load flexibility models using proposed feature $\alpha_{u}$ (red) and OAT (blue) for Large Office building

this minimum loading state using Equation 1 and Equation 2. This represents a minimum system state independent of internal and external loading conditions. The second assumption is that load shedding potential $(\Delta P)$ is proportional to the difference between the current thermal loading state $(\alpha(t))$ and the minimum loading state $(\bar{\alpha})$. We refer to this variable as the thermal unloading potential $\left(\alpha_{u}(t)\right)$. Finally, from both of these assumptions, we propose to use a linear regression model that uses $\alpha_{\mathcal{u}}(t)$ as a predictor variable to estimate the load shedding potential as shown in Equation 3.

$$
\Delta P(t)=\beta_{1} \alpha_{u}(t)+\beta_{0}
$$

\section{EXPERIMENTS}

We evaluate the proposed model using three DOE Prototype Buildings served by VAV systems: Small, Medium, and Large Office [7] and the co-simulation environment MLE+ [4]. Office buildings constitute one of the largest portions of commercial building floor space by category [8], but this approach applies generally to buildings served by VAV systems. We generated load shedding events actuated through $\mathrm{a}+2^{\circ} \mathrm{F}$ GTR for a 1 hour duration for every hour between the occupied hours of 8:00 and 19:00. The evaluation period is selected as 150 summer days from June through October excluding weekends and holidays. Demand flexibility is most valuable during the daytime in the summer [11], and the largest potential aligns with this period due to the electrical demand of chillers. This setup was repeated for five weather scenarios Miami, FL / Phoenix, AZ / San Francisco, CA / Baltimore, MD / Chicago, IL to capture distinct climate zones [2]. This yielded a total of over 1000 events per building per weather scenario. The DF potential was calculated by recording the difference between the average baseline power consumption and the average modified power consumption for the duration of the event. For every event, the thermal unloading potential and OAT were recorded immediately before the GTR was applied. Lastly, we fit linear regression models to compare the performance of both predictor variables.

\section{RESULTS}

The simulation results confirmed that the proposed feature $\left(\alpha_{u}\right)$ has better performance when predicting load shedding events when compared to OAT models. Figure 2 shows the regression models plotted for both predictor variables for the Large Office building for three weather scenarios. The results corroborate that the modeling relationship between $\alpha_{u}$ and $\Delta P$ with linear regression is appropriate. Furthermore, we can observe that a positive correlation between OAT and $\triangle P$ holds. However, as shown in the figure, several events are insensitive to OAT given that the building is already operating close to $\bar{\alpha}$. This is evidence of the limitations of a model not including information about the states in the mechanical system. In contrast, our model accurately predicts low to no shedding potential events when $\alpha$ is close to $\bar{\alpha}$. This is an often overlooked challenge when predicting the demand flexibility of oversized systems and during periods with low occupancy. To evaluate both models we calculated the root mean squared error (RMSE) and R-squared values $\left(R^{2}\right)$. Table 1 summarizes the results for all models and weather scenarios. The proposed feature has on average $55 \%$ lower RMSE and an improved $R^{2}$ across all scenarios. The performance gap is more significant for hotter climates $(77 \%$ 
Table 1: Comparison Metrics for predicting load shedding events using proposed $\alpha_{u}$ and $O A T$ features

\begin{tabular}{|c|c|c|c|c|c|c|c|c|c|c|c|c|c|}
\hline \multirow{3}{*}{ City, State } & \multirow{3}{*}{$\begin{array}{c}\text { Climate } \\
\text { Zone }\end{array}$} & \multicolumn{4}{|c|}{ Small Office } & \multicolumn{4}{|c|}{ Medium Office } & \multicolumn{4}{|c|}{ Large Office } \\
\hline & & \multicolumn{2}{|c|}{$\alpha_{u}$-Feature } & \multicolumn{2}{|c|}{$O A T$-Feature } & \multicolumn{2}{|c|}{$\alpha_{u}$-Feature } & \multicolumn{2}{|c|}{$O A T$-Feature } & \multicolumn{2}{|c|}{$\alpha_{u}$-Feature } & \multicolumn{2}{|c|}{$O A T$-Feature } \\
\hline & & RMSE & $\mathrm{R}^{2}$ & RMSE & $\mathrm{R}^{2}$ & RMSE & $\mathrm{R}^{2}$ & RMSE & $\mathrm{R}^{2}$ & RMSE & $\mathrm{R}^{2}$ & RMSE & $\mathrm{R}^{2}$ \\
\hline Miami, FL & $1 \mathrm{~A}$ & 0.26 & 0.94 & 0.90 & 0.33 & 2.18 & 0.97 & 10.42 & 0.30 & 16.02 & 0.97 & 82.85 & 0.19 \\
\hline Phoenix, AZ & $2 \mathrm{~B}$ & 0.33 & 0.85 & 0.74 & 0.23 & 4.79 & 0.88 & 12.54 & 0.20 & 27.16 & 0.92 & 94.35 & 0.08 \\
\hline San Francisco, CA & $3 \mathrm{C}$ & 0.18 & 0.80 & 0.31 & 0.38 & 2.84 & 0.77 & 3.59 & 0.64 & 25.38 & 0.72 & 30.77 & 0.59 \\
\hline Baltimore, MD & $4 \mathrm{~A}$ & 0.31 & 0.91 & 0.75 & 0.44 & 4.73 & 0.87 & 8.79 & 0.54 & 30.79 & 0.88 & 71.72 & 0.36 \\
\hline Chicago, IL & $5 \mathrm{~A}$ & 0.32 & 0.90 & 0.76 & 0.43 & 5.01 & 0.86 & 9.44 & 0.50 & 34.66 & 0.86 & 74.19 & 0.37 \\
\hline
\end{tabular}

lower RMSE in Zone $1 \mathrm{~A})$ when compared to milder climates $(27 \%$ lower RMSE in Zone 3C).

\section{CONCLUSIONS}

We proposed a scalable load flexibility model for commercial buildings with VAV systems that incorporates internal HVAC system states to predict the magnitude of load shedding events. Our approach uses terminal damper positions as a proxy to generate a single state variable, thermal unloading potential, that we use as a regressor for a linear model that estimates load shedding potential. We validated this approach through simulation experiments that demonstrated a 55\% reduction in prediction error. A limitation of this approach is that it requires access to all terminal damper positions through an advanced building automation system (BAS) while OAT models remain more ubiquitous. Another limitation is that this approach is tied to the choice of control input $\left(+2^{\circ} \mathrm{F}\right.$ GTR), and in some cases building managers can have some reservations in applying this coarse control. Finally, both OAT models and our model rely on historical event records to learn parameters, which in some cases might not be available. In future work, we plan to incorporate stochastic models for occupancy and internal loads to test the robustness of this feature. In addition, we plan to run experiments on a real testbed with multiple HVAC systems to validate the results behind the proposed feature. Furthermore, given that the proposed model does not eliminate the need for identification experiments, we plan to explore methods to initialize the model parameters using historical undisturbed data.

\section{ACKNOWLEDGMENTS}

This material is based upon work partially funded by ENGIE Lab CRIGEN, (CSAI).

\section{REFERENCES}

[1] Peter Alstone, Jennifer Potter, Mary Ann Piette, Peter Schwartz, and Michael A Berger. 2017. 2025 California Demand Response Potential Study-Charting California's Demand Response Future. Technical Report March. Lawrence Berkeley National Laboratory.

[2] ASHRAE. 2019. ANSI/ASHRAE/IES Standard 90.1-2019 Energy Standard for Buildings Except Low-Rise Residential Buildings.

[3] Benjamin Bayer. 2015. Current Practice and Thinking with Integrating Demand Response for Power System Flexibility in the Electricity Markets in the USA and Germany. Current Sustainable/Renewable Energy Reports 2, 2 (6 2015), 55-62.

[4] Willy Bernal, Madhur Behl, Truong Nghiem, and Rahul Mangharam. 2013. MLE+ ACM SIGBED Review 10, 2 (7 2013), 34-34.

[5] David H. Blum and Leslie K. Norford. 2014. Dynamic simulation and analysis of ancillary service demand response strategies for variable air volume HVAC systems. HVAC and R Research 20, 8 (11 2014), 908-921.

[6] CAISO. 2021. Demand Response Issues and Performance. , 33 pages.
[7] DOE. 2010. Commercial Prototype Building Models.

[8] EIA. 2015. Commercial Buildings Energy Consumption Survey (CBECS) 2012

[9] ERCOT. 2014. Protocol Definition for Contingency Reserve (CR) Service.

[10] Gary Goddard, Joseph Klose, and Scott Backhaus. 2014. Model Development and Identification for Fast Demand Response in Commercial HVAC Systems. IEEE Transactions on Smart Grid 5, 4 (7 2014), 2084-2092.

[11] Sen Huang, Yunyang Ye, Di Wu, and Wangda Zuo. 2021. An assessment of power flexibility from commercial building cooling systems in the United States. Energy 221 (4 2021), 119571.

[12] L.A. Hurtado, J.D. Rhodes, P.H. Nguyen, I.G. Kamphuis, and M.E. Webber. 2017. Quantifying demand flexibility based on structural thermal storage and comfort management of non-residential buildings: A comparison between hot and cold climate zones. Applied Energy 195 (6 2017), 1047-1054.

[13] Jingjing Liu, Rongxin Yin, Marco Pritoni, and Mary Ann Piette. 2020. Developing and Evaluating Metrics for Demand Flexibility in Buildings : Comparing Simulations and Field Data. In ACEEE 2020 Summer Study on Energy Efficiency in Buildings. Energy Technologies Area, 267-280.

[14] Miriam L. Goldberg and G. Kennedy Agnew. 2013. Measurement and Verification for Demand Response National Forum of the National Action Plan on Demand Response. Technical Report. LBNL.

[15] N Motegi, MA Piette, and DS Watson. 2007. Introduction to commercial building control strategies and techniques for demand response. Technical Report. Demand Response Research Center. 1-107 pages.

[16] Monica Neukomm, Valerie Nubbe, and Fares. 2019. Grid-interactive Efficient Buildings Technical Report Series: Overview of Research Challenges and Gaps. Technical Report December. US Department of Energy.

[17] Xiufeng Pang, Mary A. Piette, and Nan Zhou. 2017. Characterizing variations in variable air volume system controls. Energy and Buildings 135 (1 2017), 166-175.

[18] Mary Ann Piette, Sila Kiliccote, and Girish Ghatikar. 2014. Field Experience with and Potential for Multi-time Scale Grid Transactions from Responsive Commercial Buildings. Technical Report. LBNL.

[19] Mary Ann Piette, David Watson, Naoya Motegi, Sila Kiliccote, and Peng Xu. 2006. Automated Critical Peak Pricing field tests: Program description and results. Technical Report April. LBNL.

[20] Phillip N Price, Nathan Addy, and Sila Kiliccote. 2015. Predictability and Persistance of Demand Response Load Shed in Buildings. Technical Report. LBNL.

[21] Tariq Samad, Edward Koch, and Petr Stluka. 2016. Automated Demand Response for Smart Buildings and Microgrids: The State of the Practice and Research Challenges. Proc. IEEE 104, 4 (2016), 726-744.

[22] Andrew J Satchwell, Mary Ann Piette, Aditya Khandekar, Jessica Granderson, Natalie Mims Frick, Ryan Hledik, Ahmad Faruqui, Long Lam, Stephanie Ross, Jesse Cohen, Kitty Wang, Daniela Urigwe, Dan Delurey, Monica Neukomm, and David Nemtzow. 2021. A National Roadmap for Grid-Interactive Efficient Buildings. Technical Report. US Department of Energy. 1-166 pages.

[23] Andreas Ulbig and Göran Andersson. 2015. Analyzing operational flexibility of electric power systems. International fournal of Electrical Power and Energy Systems 72 (2015), 155-164.

[24] U.S. Energy Information Administration. 2021. Annual Energy Outlook 2021 with projections to 2050. Technical Report. US EIA, Washington, D.C.

[25] Elvin Vindel, Mario Berges, and Burcu Akinci. 2021. A Modeling Approach to Characterize the Demand Flexibility of the Air Distribution System in Commercial Buildings. In 6th International High Performance Buildings Conference at Purdue. Purdue e-Pubs, 1-10.

[26] Rongxin Yin, Emre C. Kara, Yaping Li, Nicholas DeForest, Ke Wang, Taiyou Yong, and Michael Stadler. 2016. Quantifying flexibility of commercial and residential loads for demand response using setpoint changes. Applied Energy 177 (2016), 149-164.

[27] Rongxin Yin, Peng Xu, and Sila Kiliccote. 2008. Auto-DR and Pre-cooling of Buildings at Tri-City Corporate Center. Technical Report November. Lawrence Berkeley National Laboratory. 